\title{
Completion and extension of operators in KreĬn spaces
}

\author{
DMYTRO BAIDIUK
}

(Presented by M.M. Malamud)

\begin{abstract}
A generalization of the well-known results of M.G. Kreln about the description of selfadjoint contractive extension of a hermitian contraction is obtained. This generalization concerns the situation, where the selfadjoint operator $A$ and extensions $\widetilde{A}$ belong to a Kreın space or a Pontryagin space and their defect operators are allowed to have a fixed number of negative eigenvalues. Also a result of Yu. L. Shmul'yan on completions of nonnegative block operators is generalized for block operators with a fixed number of negative eigenvalues in a Kreı̆n space.

This paper is a natural continuation of S. Hassi's and author's recent paper [7].
\end{abstract}

2010 MSC. 46C20, 47A20, 47A63.

Key words and phrases. Completion, extension of operators, Krĕn and Pontryagin spaces.

\section{Introduction}

Let $A$ be a densely defined lower semi-bounded operator in a separable Hilbert space $\mathfrak{H}, A \geq m_{A} I$. A problem of existing of selfadjoint extensions preserving the lower bound $m_{A}$ of $A$ was formulated by J. von Neumann [4]. He solved it for the case of an operator with finite deficiency indices. A solution to this problem for operators with arbitrary deficiency indices was obtained by M. Stone, H. Freudental, and K. Friedrichs [4]. M. G. Kreın in his seminal paper [19] (see also [1]) described the set $\operatorname{Ext}_{A}(0, \infty)$ of all nonnegative selfadjoint extensions $\widetilde{A}$ of $A \geq 0$ as follows

$$
\left(A_{F}+a\right)^{-1} \leq(\widetilde{A}+a)^{-1} \leq\left(A_{K}+a\right)^{-1}, \quad a>0, \quad \widetilde{A} \in \operatorname{Ext}_{A}(0, \infty) .
$$

Received 27.12.2016

The author thanks his supervisor Seppo Hassi for several detailed discussions on the results of this paper and also Mark Malamud for comments. 
Here $A_{F}$ and $A_{K}$ are the Friedrichs (hard) and Kreĭn (soft) extensions of $A$, respectively.

To obtain such a description he used a special form of the Cayley transform

$$
T_{1}=(I-A)(I+A)^{-1}, \quad T=(I-\widetilde{A})(I+\widetilde{A})^{-1},
$$

to reduce the study of unbounded operators to the study of contractive selfadjoint extensions $T$ of a Hermitian nondensely defined contraction $T_{1} \in\left[\mathfrak{H}_{1}, \mathfrak{H}\right]$, where $\mathfrak{H}_{1}=\operatorname{ran}(I+A)$. The set of all selfadjoint contractive extensions of $T_{1}$ is denoted by $\operatorname{Ext}_{T_{1}}(-1,1)$. M.G. Krel̆n proved that the set $\operatorname{Ext}_{T_{1}}(-1,1)$ forms an operator interval with minimal and maximal entries $T_{m}$ and $T_{M}$, respectively,

$$
T_{m} \leq T \leq T_{M}, \quad T \in \operatorname{Ext}_{T_{1}}(-1,1) .
$$

T. Ando and K. Nishio [2] extended main results of the Kreĭn theory to the case of nondensely defined symmetric operators $A$. For the case of linear relations (multivalued linear operators) $A \geq 0$ it was done by E.A. Coddington and H.S.V. de Snoo [9].

With respect to the orthogonal decomposition $\mathfrak{H}=\mathfrak{H}_{1} \oplus \mathfrak{H}_{2}$ a contraction $T_{1} \in\left[\mathfrak{H}_{1}, \mathfrak{H}\right]$ admits a block-matrix representation $T_{1}=\left(\begin{array}{l}T_{11} \\ T_{21}\end{array}\right)$. Block matrix representations of the operators $T_{m}$ and $T_{M}$ were obtained in $[6,18]$, and [16], (see also $[4,12,13,27]$ Namely, it is shown that

$$
\begin{aligned}
T_{m} & =\left(\begin{array}{cc}
T_{11} & D_{T_{11}} V^{*} \\
V D_{T_{11}} & -I+V\left(I-T_{11}\right) V^{*}
\end{array}\right), \\
T_{M} & =\left(\begin{array}{cc}
T_{11} & D_{T_{11}} V^{*} \\
V D_{T_{11}} & I-V\left(I+T_{11}\right) V^{*}
\end{array}\right),
\end{aligned}
$$

where $D_{T_{11}}:=\left(I-T_{11}^{2}\right)^{1 / 2}$ and $V$ is given by $V:=\operatorname{clos}\left(T_{21} D_{T_{11}}^{[-1]}\right)$. Based on these formulas a complete parametrization of the set $\operatorname{Ext}_{T_{1}}(-1,1)$ as well as main results of the Kreln theory have also been obtained there. In turn, the proof of formulas for $T_{m}$ and $T_{M}$ was based on a result of Yu. L. Shmul'yan [26] (see also [27]) of nonnegative completions of a nonnegative block operator.

Recently, S. Hassi and the author [7] extended the main result of [16] to the case of "quasi-contractive" symmetric operators $T_{1}$. Recall, that the "quasi-contractivity" means that $\nu_{-}\left(I-T^{*} T\right)<\infty$, where

$$
\nu_{-}(K)=\operatorname{dim}\left(E_{K}(-\infty, 0) \mathfrak{H}\right) .
$$

For this purpose the above mentioned result of Shmul'yan was generalized there. Also an analog of block matrix formulas for the operators $T_{m}$ and 
$T_{M}$ was established. Formulas $T_{m}$ and $T_{M}$ in this case look similar to (1.1) but the entries $V\left(I \pm T_{11}\right) V^{*}$ are replaced by $V\left(I \pm T_{11}\right) J V^{*}$, where $J=\operatorname{sign}\left(I-T_{11}^{2}\right)$ and $D_{T_{11}}:=\left|I-T_{11}^{2}\right|^{1 / 2}$.

The first result of the present paper is a further generalization of Shmul'yan's result [26] to the case of block operators acting in a KreĬn space and having a fixed number of negative eigenvalues.

In Section 4 a first Kreln space analog of completion problem is formulated and a description of its solutions is found. Namely, we consider classes of "quasi-contractive" symmetric operators $T_{1}$ in a Kreun space with $\nu_{-}\left(I-T_{1}^{*} T_{1}\right)<\infty$ and describe all possible selfadjoint (in the Krĕn space sense) extensions $T$ of $T_{1}$ which preserve the given negative index $\nu_{-}\left(I-T^{*} T\right)=\nu_{-}\left(I-T_{1}^{*} T_{1}\right)$. This problem is close to the completion problem studied in [7] and has a similar description for its solutions. For related problems see also [3-5, 10-16, 18, 20, 22-25, 27].

The main result of the present paper is Theorem 5.7. Namely, we consider classes of "quasi-contractive" symmetric operators $T_{1}$ in a Pontryagin space $(\mathfrak{H}, J)$ with

$$
\nu_{-}\left[I-T_{1}^{[*]} T_{1}\right]:=\nu_{-}\left(J\left(I-T_{1}^{[*]} T_{1}\right)\right)<\infty
$$

and we establish a solvability criterion and a description of all possible selfadjoint extensions $T$ of $T_{1}$ (in the Pontryagin space sense) which preserve the given negative index $\nu_{-}\left[I-T^{[*]} T\right]=\nu_{-}\left[I-T_{1}^{[*]} T_{1}\right]$. The formulas for $T_{m}$ and $T_{M}$ are also extended in an appropriate manner (see (5.16)). It should be emphasized that in this more general setting formulas (5.16) involve so-called link operator $L_{T}$ which was introduced by Arsene, Constantintscu and Gheondea in [5] (see also [4,10,11,21]).

\section{A completion problem for block operators in Kreŭn spaces}

By definition the modulus $|C|$ of a closed operator $C$ is the nonnegative selfadjoint operator $|C|=\left(C^{*} C\right)^{1 / 2}$. Every closed operator admits a polar decomposition $C=U|C|$, where $U$ is a (unique) partial isometry with the initial space $\overline{\mathrm{ran}}|C|$ and the final space $\overline{\mathrm{ran}} C$, cf. [17]. For a selfadjoint operator $H=\int_{\mathbb{R}} t d E_{t}$ in a Hilbert space $\mathfrak{H}$ the partial isometry $U$ can be identified with the signature operator, which can be taken to be unitary: $J=\operatorname{sign}(H)=\int_{\mathbb{R}} \operatorname{sign}(t) d E_{t}$, in which case one should define $\operatorname{sign}(t)=1$ if $t \geq 0$ and otherwise $\operatorname{sign}(t)=-1$.

Let $\mathcal{H}$ be a Hilbert space, and let $J_{\mathcal{H}}$ be a signature operator in it, i.e., $J_{\mathcal{H}}=J_{\mathcal{H}}^{*}=J_{\mathcal{H}}^{-1}$. We interpret the space $\mathcal{H}$ as a $\operatorname{Kreun~space~}\left(\mathcal{H}, J_{\mathcal{H}}\right)$ 
(see $[6,8]$ ) in which the indefinite scalar product is defined by the equality

$$
[\varphi, \psi]_{\mathcal{H}}=\left(J_{\mathcal{H}} \varphi, \psi\right)_{\mathcal{H}}
$$

Let us introduce a partial ordering for selfadjoint Kreĭn space operators. For selfadjoint operators $A$ and $B$ with the same domains $A \geq_{J} B$ if and only if $[(A-B) f, f] \geq 0$ for all $f \in \operatorname{dom} A$. If not otherwise indicated the word "smallest" means the smallest operator in the sense of this partial ordering.

Consider a bounded incomplete block operator

$$
A^{0}=\left(\begin{array}{cc}
A_{11} & A_{12} \\
A_{21} & *
\end{array}\right)\left(\begin{array}{c}
\left(\mathfrak{H}_{1}, J_{1}\right) \\
\left(\mathfrak{H}_{2}, J_{2}\right)
\end{array}\right) \rightarrow\left(\begin{array}{l}
\left(\mathfrak{H}_{1}, J_{1}\right) \\
\left(\mathfrak{H}_{2}, J_{2}\right)
\end{array}\right)
$$

in the Kre ̌n space $\mathfrak{H}=\left(\mathfrak{H}_{1} \oplus \mathfrak{H}_{2}, J\right)$, where $\left(\mathfrak{H}_{1}, J_{1}\right)$ and $\left(\mathfrak{H}_{2}, J_{2}\right)$ are Kreı̆n spaces with fundamental symmetries $J_{1}$ and $J_{2}$, and $J=\left(\begin{array}{cc}J_{1} & 0 \\ 0 & J_{2}\end{array}\right)$.

Theorem 2.1. Let $\mathfrak{H}=\left(\mathfrak{H}_{1} \oplus \mathfrak{H}_{2}, J\right)$ be an orthogonal decomposition of the Krein space $\mathfrak{H}$ and let $A^{0}$ be an incomplete block operator of the form (2.1). Assume that $A_{11}=A_{11}^{[*]}$ and $A_{21}=A_{12}^{[*]}$ are bounded, the numbers of negative squares of the quadratic form $\left[A_{11} f, f\right]\left(f \in \operatorname{dom} A_{11}\right)$ $\nu_{-}\left[A_{11}\right]:=\nu_{-}\left(J_{1} A_{11}\right)=\kappa<\infty$, where $\kappa \in \mathbb{Z}_{+}$, and let us introduce $J_{11}:=\operatorname{sign}\left(J_{1} A_{11}\right)$ the (unitary) signature operator of $J_{1} A_{11}$. Then:

(i) There exists a completion $A \in[(\mathfrak{H}, J)]$ of $A^{0}$ with some operator $A_{22}=A_{22}^{[*]} \in\left[\left(\mathfrak{H}_{2}, J_{2}\right)\right]$ such that $\nu_{-}[A]=\nu_{-}\left[A_{11}\right]=\kappa$ if and only if

$$
\operatorname{ran} J_{1} A_{12} \subset \operatorname{ran}\left|A_{11}\right|^{1 / 2} .
$$

(ii) In this case the operator $S=\left|A_{11}\right|^{[-1 / 2]} J_{1} A_{12}$, where $\left|A_{11}\right|^{[-1 / 2]}$ denotes the (generalized) Moore-Penrose inverse of $\left|A_{11}\right|^{1 / 2}$, is well defined and $S \in\left[\left(\mathfrak{H}_{2}, J_{2}\right),\left(\mathfrak{H}_{1}, J_{1}\right)\right]$. Moreover, $S^{[*]} J_{1} J_{11} S$ is the "smallest" operator in the solution set

$$
\mathcal{A}:=\left\{A_{22}=A_{22}^{[*]} \in\left[\left(\mathfrak{H}_{2}, J_{2}\right)\right]: A=\left(A_{i j}\right)_{i, j=1}^{2}: \nu_{-}[A]=\kappa\right\}
$$

and this solution set admits a description

$$
\begin{aligned}
\mathcal{A}= & \left\{A_{22} \in\left[\left(\mathfrak{H}_{2}, J_{2}\right)\right]: A_{22}=J_{2}\left(S^{*} J_{11} S+Y\right)=S^{[*]} J_{1} J_{11} S+J_{2} Y,\right. \\
& \text { where } \left.Y=Y^{*} \geq 0\right\} .
\end{aligned}
$$


Proof. Let us introduce a block operator

$$
\widetilde{A}^{0}=\left(\begin{array}{cc}
\widetilde{A}_{11} & \widetilde{A}_{12} \\
\widetilde{A}_{21} & *
\end{array}\right)=\left(\begin{array}{cc}
J_{1} A_{11} & J_{1} A_{12} \\
J_{2} A_{21} & *
\end{array}\right) .
$$

The blocks of this operator satisfy the identities $\widetilde{A}_{11}=\widetilde{A}_{11}^{*}, \widetilde{A}_{21}^{*}=\widetilde{A}_{12}$ and

$$
\begin{aligned}
\operatorname{ran} J_{1} A_{11} & =\operatorname{ran} \widetilde{A}_{11} \subset \operatorname{ran}\left|\widetilde{A}_{11}\right|^{1 / 2}=\operatorname{ran}\left(\widetilde{A}_{11}^{*} \widetilde{A}_{11}\right)^{1 / 4} \\
& =\operatorname{ran}\left(A_{11}^{*} A_{11}\right)^{1 / 4}=\operatorname{ran}\left|A_{11}\right|^{1 / 2} .
\end{aligned}
$$

Then due to [7, Theorem 1] a description of all selfadjoint operator completions of $\widetilde{A}^{0}$ admits representation $\widetilde{A}=\left(\begin{array}{ll}\widetilde{A}_{11} & \widetilde{A}_{12} \\ \widetilde{A}_{21} & \widetilde{A}_{22}\end{array}\right)$ with $\widetilde{A}_{22}=$ $\widetilde{S}^{*} J_{11} \widetilde{S}+Y$, where $\widetilde{S}=\left|\widetilde{A}_{11}\right|^{[-1 / 2]} \widetilde{A}_{12}$ and $Y=Y^{*} \geq 0$.

This yields description for the solutions of the completion problem. The set of completions has the form $A=\left(\begin{array}{ll}A_{11} & A_{12} \\ A_{21} & A_{22}\end{array}\right)$, where

$$
\begin{aligned}
A_{22} & =J_{2} \widetilde{A}_{22}=J_{2} A_{21} J_{1}\left|A_{11}\right|^{[-1 / 2]} J_{11}\left|A_{11}\right|^{[-1 / 2]} J_{1} A_{12}+J_{2} Y \\
& =J_{2} S^{*} J_{11} S+J_{2} Y=S^{[*]} J_{1} J_{11} S+J_{2} Y .
\end{aligned}
$$

\section{Some inertia formulas}

Some simple inertia formulas are now recalled. The factorization $H=$ $B^{[*]} E B$ clearly implies that $\nu_{ \pm}[H] \leq \nu_{ \pm}[E]$, cf. (1.2). If $H_{1}$ and $H_{2}$ are selfadjoint operators in a Kreı̆n space, then

$$
H_{1}+H_{2}=\left(\begin{array}{l}
I \\
I
\end{array}\right)^{[*]}\left(\begin{array}{cc}
H_{1} & 0 \\
0 & H_{2}
\end{array}\right)\left(\begin{array}{l}
I \\
I
\end{array}\right)
$$

shows that $\nu_{ \pm}\left[H_{1}+H_{2}\right] \leq \nu_{ \pm}\left[H_{1}\right]+\nu_{ \pm}\left[H_{2}\right]$. Consider the selfadjoint block operator $H \in\left[\left(\mathfrak{H}_{1}, J_{1}\right) \oplus\left(\mathfrak{H}_{2}, J_{2}\right)\right]$, where $J_{i}=J_{i}^{*}=J_{i}^{-1},(i=1,2)$ of the form

$$
H=H^{[*]}=\left(\begin{array}{cc}
A & B^{[*]} \\
B & I
\end{array}\right)
$$

By applying the above mentioned inequalities shows that

$$
\nu_{ \pm}[A] \leq \nu_{ \pm}\left[A-B^{[*]} B\right]+\nu_{ \pm}\left(J_{2}\right) .
$$

Assuming that $\nu_{-}\left[A-B^{*} J_{2} B\right]$ and $\nu_{-}\left(J_{2}\right)$ are finite, the question when $\nu_{-}[A]$ attains its maximum in (3.1), or equivalently, $\nu_{-}\left[A-B^{*} J_{2} B\right] \geq$ 
$\nu_{-}[A]-\nu_{-}\left(J_{2}\right)$ attains its minimum, turns out to be of particular interest. The next result characterizes this situation as an application of Theorem 2.1. Recall that if $J_{1} A=J_{A}|A|$ is the polar decomposition of $J_{1} A$, then one can interpret $\mathfrak{H}_{A}=\left(\overline{\mathrm{ran}} J_{1} A, J_{A}\right)$ as a Krel̆n space generated on $\overline{\operatorname{ran}} J_{1} A$ by the fundamental symmetry $J_{A}=\operatorname{sign}\left(J_{1} A\right)$.

Theorem 3.1. Let $A \in\left[\left(\mathfrak{H}_{1}, J_{1}\right)\right]$ be selfadjoint, $B \in\left[\left(\mathfrak{H}_{1}, J_{1}\right),\left(\mathfrak{H}_{2}, J_{2}\right)\right]$, $J_{i}=J_{i}^{*}=J_{i}^{-1} \in\left[\mathfrak{H}_{i}\right],(i=1,2)$, and assume that $\nu_{-}[A], \nu_{-}\left(J_{2}\right)<\infty$. If the equality

$$
\nu_{-}[A]=\nu_{-}\left[A-B^{[*]} B\right]+\nu_{-}\left(J_{2}\right)
$$

holds, then $\operatorname{ran} J_{1} B^{[*]} \subset \operatorname{ran}|A|^{1 / 2}$ and $J_{1} B^{[*]}=|A|^{1 / 2} K$ for a unique operator $K \in\left[\left(\mathfrak{H}_{2}, J_{2}\right), \mathfrak{H}_{A}\right]$ which is $J$-contractive: $J_{2}-K^{*} J_{A} K \geq 0$.

Conversely, if $B^{[*]}=|A|^{1 / 2} K$ for some $J$-contractive operator $K \in$ $\left[\left(\mathfrak{H}_{2}, J_{2}\right), \mathfrak{H}_{A}\right]$, then the equality (3.1) is satisfied.

Proof. Assume that (3.1) is satisfied. The factorization

$$
H=\left(\begin{array}{cc}
A & B^{[*]} \\
B & I
\end{array}\right)=\left(\begin{array}{cc}
I & B^{[*]} \\
0 & I
\end{array}\right)\left(\begin{array}{cc}
A-B^{[*]} B & 0 \\
0 & I
\end{array}\right)\left(\begin{array}{cc}
I & 0 \\
B & I
\end{array}\right)
$$

shows that $\nu_{-}[H]=\nu_{-}\left[A-B^{[*]} B\right]+\nu_{-}\left(J_{2}\right)$, which combined with the equality (3.1) gives $\nu_{-}[H]=\nu_{-}[A]$. Therefore, by Theorem 2.1 one has $\operatorname{ran} J_{1} B^{[*]} \subset \operatorname{ran}|A|^{1 / 2}$ and this is equivalent to the existence of a unique operator $K \in\left[\left(\mathfrak{H}_{2}, J_{2}\right), \mathfrak{H}_{A}\right]$ such that $J_{1} B^{[*]}=|A|^{1 / 2} K$; i.e. $K=|A|^{[-1 / 2]} J_{1} B^{[*]}$. Furthermore, $K^{[*]} J_{1} J_{A} K \leq_{J_{2}} I$ by the minimality property of $K^{[*]} J_{1} J_{A} K$ in Theorem 2.1 , in other words $K$ is a $J$ contraction.

Converse, if $J_{1} B^{[*]}=|A|^{1 / 2} K$ for some $J$-contractive operator $K \in$ $\left[\left(\mathfrak{H}_{2}, J_{2}\right), \mathfrak{H}_{A}\right]$, then clearly $\operatorname{ran} J_{1} B^{[*]} \subset \operatorname{ran}|A|^{1 / 2}$. By Theorem 2.1 the completion problem for $H^{0}$ has solutions with the minimal solution $S^{[*]} J_{1} J_{A} S$, where

$$
S=|A|^{[-1 / 2]} J_{1} B^{[*]}=|A|^{[-1 / 2]}|A|^{1 / 2} K=K .
$$

Furthermore, by $J$-contractivity of $K$ one has $K^{[*]} J_{1} J_{A} K \leq_{J_{2}} I$, i.e. $I$ is also a solution and thus $\nu_{-}[H]=\nu_{-}[A]$ or, equivalently, the equality (3.1) is satisfied.

\section{A pair of completion problems in a Krel̆n space}

In this section we introduce and describe the solutions of a Krein space version of a completion problem that was treated in [7]. 
Let $\left(\mathfrak{H}_{i},\left(J_{i}, \cdot\right)\right)$ and $(\mathfrak{H},(J \cdot, \cdot))$ be Krĕn spaces, where $\mathfrak{H}=\mathfrak{H}_{1} \oplus$ $\mathfrak{H}_{2}, J=\left(\begin{array}{cc}J_{1} & 0 \\ 0 & J_{2}\end{array}\right)$, and $J_{i}$ are fundamental symmetries $(i=1,2)$, let $T_{11}=T_{11}^{[*]} \in\left[\left(\mathfrak{H}_{1}, J_{1}\right)\right]$ be an operator such that $\nu_{-}\left(I-T_{11}^{*} T_{11}\right)=\kappa<\infty$. Denote $\widetilde{T}_{11}=J_{1} T_{11}$, then $\widetilde{T}_{11}=\widetilde{T}_{11}^{*}$ in the Hilbert space $\mathfrak{H}_{1}$. Rewrite $\nu_{-}\left(I-T_{11}^{*} T_{11}\right)=\nu_{-}\left(I-\widetilde{T}_{11}^{2}\right)$. Denote

$$
J_{+}=\operatorname{sign}\left(I-\widetilde{T}_{11}\right), J_{-}=\operatorname{sign}\left(I+\widetilde{T}_{11}\right), \text { and } J_{11}=\operatorname{sign}\left(I-\widetilde{T}_{11}^{2}\right),
$$

and let $\kappa_{+}=\nu_{-}\left(J_{+}\right)$and $\kappa_{-}=\nu_{-}\left(J_{-}\right)$. It is easy to get that $J_{11}=$ $J_{-} J_{+}=J_{+} J_{-}$. Moreover, there is an equality $\kappa=\kappa_{-}+\kappa_{+}$(see [7, Lemma 5.1]). We recall the results for the operator $\widetilde{T}_{11}$ from the paper [7] and after that reformulate them for the operator $T_{11}$. We recall completion problem and its solutions that was investigated in a Hilbert space setting in [7]. The problem concerns the existence and a description of selfadjoint operators $\widetilde{T}$ such that $\widetilde{A}_{+}=I+\widetilde{T}$ and $\widetilde{A}_{-}=I-\widetilde{T}$ solve the corresponding completion problems

$$
\widetilde{A}_{ \pm}^{0}=\left(\begin{array}{cc}
I \pm \widetilde{T}_{11} & \pm \widetilde{T}_{21}^{*} \\
\pm \widetilde{T}_{21} & *
\end{array}\right)
$$

under minimal index conditions $\nu_{-}(I+\widetilde{T})=\nu_{-}\left(I+\widetilde{T}_{11}\right), \nu_{-}(I-\widetilde{T})=$ $\nu_{-}\left(I-\widetilde{T}_{11}\right)$, respectively. The solution set is denoted by $\operatorname{Ext}_{\widetilde{T}_{1}, \kappa}(-1,1)$.

The next theorem gives a general solvability criterion for the completion problem (4.1) and describes all solutions to this problem.

Theorem 4.1. ([7, Theorem 5]) Let $\widetilde{T}_{1}=\left(\begin{array}{l}\widetilde{T}_{11} \\ \widetilde{T}_{21}\end{array}\right): \mathfrak{H}_{1} \rightarrow\left(\begin{array}{l}\mathfrak{H}_{1} \\ \mathfrak{H}_{2}\end{array}\right)$ be a symmetric operator with $\widetilde{T}_{11}=\widetilde{T}_{11}^{*} \in\left[\mathfrak{H}_{1}\right]$ and $\nu_{-}\left(I-\widetilde{T}_{11}^{2}\right)=\kappa<\infty$, and let $J_{11}=\operatorname{sign}\left(I-\widetilde{T}_{11}^{2}\right)$. Then the completion problem for $\widetilde{A}_{ \pm}^{0}$ in $(4.1)$ has a solution $I \pm \widetilde{T}$ for some $\widetilde{T}=\widetilde{T}^{*}$ with $\nu_{-}\left(I-\widetilde{T}^{2}\right)=\kappa$ if and only if the following condition is satisfied:

$$
\nu_{-}\left(I-\widetilde{T}_{11}^{2}\right)=\nu_{-}\left(I-\widetilde{T}_{1}^{*} \widetilde{T}_{1}\right)
$$

If this condition is satisfied then the following facts hold:

(i) The completion problems for $\widetilde{A}_{ \pm}^{0}$ in (4.1) have minimal solutions $\widetilde{A}_{ \pm}$.

(ii) The operators $\widetilde{T}_{m}:=\widetilde{A}_{+}-I$ and $\widetilde{T}_{M}:=I-\widetilde{A}_{-} \in \operatorname{Ext}_{\widetilde{T}_{1}, \kappa}(-1,1)$. 
(iii) The operators $\widetilde{T}_{m}$ and $\widetilde{T}_{M}$ have the block form

$$
\begin{gathered}
\widetilde{T}_{m}=\left(\begin{array}{cc}
\widetilde{T}_{11} & D_{\widetilde{T}_{11}} V^{*} \\
V D_{\widetilde{T}_{11}} & -I+V\left(I-\widetilde{T}_{11}\right) J_{11} V^{*}
\end{array}\right), \\
\widetilde{T}_{M}=\left(\begin{array}{cc}
\widetilde{T}_{11} & D_{\widetilde{T}_{11}} V^{*} \\
V \widetilde{T}_{\widetilde{T}_{11}} & I-V\left(I+\widetilde{T}_{11}\right) J_{11} V^{*}
\end{array}\right),
\end{gathered}
$$

where $D_{\widetilde{T}_{11}}:=\left|I-\widetilde{T}_{11}^{2}\right|^{1 / 2}$ and $V$ is given by $V:=\operatorname{clos}\left(\widetilde{T}_{21} D_{\widetilde{T}_{11}}^{[-1]}\right)$.

(iv) The operators $\widetilde{T}_{m}$ and $\widetilde{T}_{M}$ are extremal extensions of $\widetilde{T}_{1}$ :

$$
\widetilde{T} \in \operatorname{Ext}_{\widetilde{T}_{1}, \kappa}(-1,1) \quad \text { iff } \widetilde{T}=\widetilde{T}^{*} \in[\mathfrak{H}], \quad \widetilde{T}_{m} \leq \widetilde{T} \leq \widetilde{T}_{M} .
$$

(v) The operators $\widetilde{T}_{m}$ and $\widetilde{T}_{M}$ are connected via

$$
(-\widetilde{T})_{m}=-\widetilde{T}_{M}, \quad(-\widetilde{T})_{M}=-\widetilde{T}_{m} .
$$

For what follows it is convenient to reformulate the above theorem in a Kreĭn space setting. Consider the Kreĭn space $(\mathfrak{H}, J)$ and a selfadjoint operator $T$ in this space. Now the problem concerns selfadjoint operators $A_{+}=I+T$ and $A_{-}=I-T$ in the Krein space $(\mathfrak{H}, J)$ that solve the completion problems

$$
A_{ \pm}^{0}=\left(\begin{array}{cc}
I \pm T_{11} & \pm T_{21}^{[*]} \\
\pm T_{21} & *
\end{array}\right),
$$

under minimal index conditions $\nu_{-}(I+J T)=\nu_{-}\left(I+J_{1} T_{11}\right)$ and $\nu_{-}(I-$ $J T)=\nu_{-}\left(I-J_{1} T_{11}\right)$, respectively. The set of solutions $T$ to the problem (4.4) will be denoted by Ext $J_{2} T_{1}, \kappa(-1,1)$.

Denote

$$
T_{1}=\left(\begin{array}{l}
T_{11} \\
T_{21}
\end{array}\right):\left(\mathfrak{H}_{1}, J_{1}\right) \rightarrow\left(\begin{array}{l}
\left(\mathfrak{H}_{1}, J_{1}\right) \\
\left(\mathfrak{H}_{2}, J_{2}\right)
\end{array}\right)
$$

so that $T_{1}$ is symmetric (nondensely defined) operator in the Kreln space $\left[\left(\mathfrak{H}_{1}, J_{1}\right)\right]$, i.e. $T_{11}=T_{11}^{[*]}$.

Theorem 4.2. Let $T_{1}$ be a symmetric operator in a Kreĭn space sense as in (4.5) with $T_{11}=T_{11}^{[*]} \in\left[\left(\mathfrak{H}_{1}, J_{1}\right)\right]$ and $\nu_{-}\left(I-T_{11}^{*} T_{11}\right)=\kappa<\infty$, and let $J=\operatorname{sign}\left(I-T_{11}^{*} T_{11}\right)$. Then the completion problems for $A_{ \pm}^{0}$ in (4.4) have a solution $I \pm T$ for some $T=T^{[*]}$ with $\nu_{-}\left(I-T^{*} T\right)=\kappa$ if and only if the following condition is satisfied:

$$
\nu_{-}\left(I-T_{11}^{*} T_{11}\right)=\nu_{-}\left(I-T_{1}^{*} T_{1}\right) .
$$

If this condition is satisfied then the following facts hold: 
(i) The completion problems for $A_{ \pm}^{0}$ in (4.4) have "minimal" ( $J_{2}-m i$ nimal) solutions $A_{ \pm}$.

(ii) The operators $T_{m}:=A_{+}-J$ and $T_{M}:=J-A_{-} \in \operatorname{Ext}_{J_{2} T_{1}, \kappa}(-1,1)$.

(iii) The operators $T_{m}$ and $T_{M}$ have the block form

$$
\begin{aligned}
& T_{m}=\left(\begin{array}{cc}
T_{11} & J_{1} D_{T_{11}} V^{*} \\
J_{2} V D_{T_{11}} & -J_{2}+J_{2} V\left(I-J_{1} T_{11}\right) J_{11} V^{*}
\end{array}\right), \\
& T_{M}=\left(\begin{array}{cc}
T_{11} & J_{1} D_{T_{11}} V^{*} \\
J_{2} V D_{T_{11}} & J_{2}-J_{2} V\left(I+J_{1} T_{11}\right) J_{11} V^{*}
\end{array}\right),
\end{aligned}
$$

where $D_{T_{11}}:=\left|I-T_{11}^{*} T_{11}\right|^{1 / 2}$ and $V$ is given by $V:=\operatorname{clos}\left(J_{2} T_{21} D_{T_{11}}^{[-1]}\right)$.

(iv) The operators $T_{m}$ and $T_{M}$ are $J_{2}$-extremal extensions of $T_{1}$ :

$T \in{\text { Ext } J_{2} T_{1}, \kappa}(-1,1) \quad$ iff $T=T^{[*]} \in[(\mathfrak{H}, J)], \quad T_{m} \leq_{J_{2}} T \leq_{J_{2}} T_{M}$.

(v) The operators $T_{m}$ and $T_{M}$ are connected via

$$
(-T)_{m}=-T_{M}, \quad(-T)_{M}=-T_{m} .
$$

Proof. The proof is obtained by systematic use of the equivalence that $T$ is a selfadjoint operator in a Kreı̆n space if and only if $\widetilde{T}$ is a selfadjoint in a Hilbert space. In particular, $T$ gives solutions to the completion problems (4.4) if and only if $\widetilde{T}$ solves the completion problems (4.4). In view of

$$
I-T_{11}^{*} T_{11}=I-T_{11}^{*} J J T_{11}=I-\widetilde{T}_{11}^{2},
$$

we are getting formula (4.6) from (4.2). Then formula (4.7) follows by multiplying the operators in (4.3) by the fundamental symmetry.

\section{Completion problem in a Pontryagin space}

\subsection{Defect operators and link operators}

Let $(\mathfrak{H},(\cdot, \cdot))$ be a Hilbert space and let $J$ be a symmetry in $\mathfrak{H}$, i.e. $J=J^{*}=J^{-1}$, so that $(\mathfrak{H},(J \cdot, \cdot))$, becomes a Pontryagin space. Then associate with $T \in[\mathfrak{H}]$ the corresponding defect and signature operators

$$
D_{T}=\left|J-T^{*} J T\right|^{1 / 2}, \quad J_{T}=\operatorname{sign}\left(J-T^{*} J T\right), \quad \mathfrak{D}_{T}=\overline{\operatorname{ran}} D_{T},
$$

where the so-called defect subspace $\mathfrak{D}_{T}$ can be considered as a Pontryagin space with the fundamental symmetry $J_{T}$. Similar notations are used with $T^{*}$ :

$$
D_{T^{*}}=\left|J-T J T^{*}\right|^{1 / 2}, \quad J_{T^{*}}=\operatorname{sign}\left(J-T J T^{*}\right), \quad \mathfrak{D}_{T^{*}}=\overline{\operatorname{ran}} D_{T^{*}} .
$$


By definition $J_{T} D_{T}^{2}=J-T^{*} J T$ and $J_{T} D_{T}=D_{T} J_{T}$ with analogous identities for $D_{T^{*}}$ and $J_{T^{*}}$. In addition,

$$
\left(J-T^{*} J T\right) J T^{*}=T^{*} J\left(J-T J T^{*}\right),\left(J-T J T^{*}\right) J T=T J\left(J-T^{*} J T\right) .
$$

Recall that $T \in[\mathfrak{H}]$ is said to be a $J$-contraction if $J-T^{*} J T \geq 0$, i.e. $\nu_{-}\left(J-T^{*} J T\right)=0$. If, in addition, $T^{*}$ is a $J$-contraction, $T$ is termed as a $J$-bicontraction.

For the following consideration an indefinite version of the commutation relation of the form $T D_{T}=D_{T^{*}} T$ is needed; these involve so-called link operators introduced in [5, Section 4] (see also [7]).

Definition 5.1. There exist unique operators $L_{T} \in\left[\mathfrak{D}_{T}, \mathfrak{D}_{T^{*}}\right]$ and $L_{T^{*}} \in$ $\left[\mathfrak{D}_{T^{*}}, \mathfrak{D}_{T}\right]$ such that

$$
D_{T^{*}} L_{T}=T J D_{T}\left\lceil\mathfrak{D}_{T}, \quad D_{T} L_{T^{*}}=T^{*} J D_{T^{*}} \uparrow \mathfrak{D}_{T^{*}} ;\right.
$$

in fact, $L_{T}=D_{T^{*}}^{[-1]} T J D_{T}\left\lceil\mathfrak{D}_{T}\right.$ and $L_{T^{*}}=D_{T}^{[-1]} T^{*} J D_{T^{*}}\left\lceil\mathfrak{D}_{T^{*}}\right.$.

The following identities can be obtained with direct calculations; see [5, Section 4]:

$$
\begin{gathered}
L_{T^{*}}^{*} J_{T^{*}}\left\lceil\mathfrak{D}_{T^{*}}=J_{T} L_{T^{*}} ;\right. \\
\left(J_{T}-D_{T} J D_{T}\right)\left\lceil\mathfrak{D}_{T}=L_{T}^{*} J_{T^{*}} L_{T} ;\right. \\
\left(J_{T^{*}}-D_{T^{*}} J D_{T^{*}}\right)\left\lceil\mathfrak{D}_{T^{*}}=L_{T^{*}}^{*} J_{T} L_{T^{*}} .\right.
\end{gathered}
$$

Now let $T$ be selfadjoint in Pontryagin space $(\mathfrak{H}, J)$, i.e. $T^{*}=J T J$. Then connections between $D_{T^{*}}$ and $D_{T}, J_{T^{*}}$ and $J_{T}, L_{T^{*}}$ and $L_{T}$ can be established.

Lemma 5.1. Assume that $T^{*}=J T J$. Then $D_{T}=\left|I-T^{2}\right|^{1 / 2}$ and the following equalities hold:

$$
D_{T^{*}}=J D_{T} J
$$

in particular,

$$
\begin{gathered}
\mathfrak{D}_{T^{*}}=J \mathfrak{D}_{T} \text { and } \mathfrak{D}_{T}=J \mathfrak{D}_{T^{*}} ; \\
J_{T^{*}}=J J_{T} J ; \\
L_{T^{*}}=J L_{T} J .
\end{gathered}
$$

Proof. The defect operator of $T$ can be calculated by the formula

$$
D_{T}=\left(\left(I-\left(T^{*}\right)^{2}\right) J J\left(I-T^{2}\right)\right)^{1 / 4}=\left(\left(I-\left(T^{*}\right)^{2}\right)\left(I-T^{2}\right)\right)^{1 / 4} .
$$


Then

$$
\begin{aligned}
D_{T^{*}} & =\left(J\left(I-\left(T^{*}\right)^{2}\right)\left(I-T^{2}\right) J\right)^{1 / 4}=J\left(\left(I-\left(T^{*}\right)^{2}\right)\left(I-T^{2}\right)\right)^{1 / 4} J \\
& =J D_{T} J
\end{aligned}
$$

i.e. (5.3) holds. This implies

$$
J \mathfrak{D}_{T^{*}} \subset \mathfrak{D}_{T} \text { and } J \mathfrak{D}_{T} \subset \mathfrak{D}_{T^{*}} .
$$

Hence from the last two formulas we get

$$
\mathfrak{D}_{T^{*}}=J\left(J \mathfrak{D}_{T^{*}}\right) \subset J \mathfrak{D}_{T} \subset \mathfrak{D}_{T^{*}}
$$

and similarly

$$
\mathfrak{D}_{T}=J\left(J \mathfrak{D}_{T}\right) \subset J \mathfrak{D}_{T^{*}} \subset \mathfrak{D}_{T}
$$

The formula

$$
\begin{aligned}
J_{T} D_{T}^{2} & =J-T^{*} J T=J\left(J-T J T^{*}\right) J=J J_{T^{*}} D_{T^{*}}^{2} J=J J_{T^{*}} J D_{T}^{2} J J \\
& =J J_{T^{*}} J D_{T}^{2}
\end{aligned}
$$

yields the equation (5.4).

The relation (5.5) follows from

$$
D_{T} L_{T^{*}}=T^{*} J D_{T^{*}} \mid \mathfrak{D}_{T^{*}}=J T J D_{T} J\left\lceil\mathfrak{D}_{T^{*}}=J D_{T^{*}} L_{T} J=D_{T} J L_{T} J .\right.
$$

\subsection{Lemmas on negative indices of certain block operators}

The first two lemmas are of preparatory nature for the last two lemmas, which are used for the proof of the main theorem.

Lemma 5.2. Let $\left(\begin{array}{cc}J & T \\ T & J\end{array}\right):\left(\begin{array}{l}\mathfrak{H} \\ \mathfrak{H}\end{array}\right) \rightarrow\left(\begin{array}{l}\mathfrak{H} \\ \mathfrak{H}\end{array}\right)$ be a selfadjoint operator in the Hilbert space $\mathfrak{H}^{2}=\mathfrak{H} \oplus \mathfrak{H}$. Then

$$
\left|\left(\begin{array}{cc}
J & T \\
T & J
\end{array}\right)\right|^{1 / 2}=U\left(\begin{array}{cc}
|J+T|^{1 / 2} & 0 \\
0 & |J-T|^{1 / 2}
\end{array}\right) U^{*}
$$

where $U=\frac{1}{\sqrt{2}}\left(\begin{array}{cc}I & I \\ I & -I\end{array}\right)$ is a unitary operator. 
Proof. It is easy to check that

$$
\left(\begin{array}{cc}
J & T \\
T & J
\end{array}\right)=U\left(\begin{array}{cc}
J+T & 0 \\
0 & J-T
\end{array}\right) U^{*}
$$

Then by taking the modulus one gets

$$
\left|\left(\begin{array}{cc}
J & T \\
T & J
\end{array}\right)\right|^{2}=\left(\left(\begin{array}{cc}
J & T \\
T & J
\end{array}\right)^{*}\left(\begin{array}{cc}
J & T \\
T & J
\end{array}\right)\right)=U\left(\begin{array}{cc}
|J+T|^{2} & 0 \\
0 & |J-T|^{2}
\end{array}\right) U^{*} .
$$

The last step is to extract the square roots (twice) from the both sides of the equation:

$$
\left|\left(\begin{array}{cc}
J & T \\
T & J
\end{array}\right)\right|^{1 / 2}=U\left(\begin{array}{cc}
|J+T|^{1 / 2} & 0 \\
0 & |J-T|^{1 / 2}
\end{array}\right) U^{*}
$$

The right hand side can be written in this form because $U$ is unitary.

Lemma 5.3. Let $T=T^{*} \in \mathfrak{H}$ be a selfadjoint operator in a Hilbert space $\mathfrak{H}$ and let $J=J^{*}=J^{-1}$ be a fundamental symmetry in $\mathfrak{H}$ with $\nu_{-}(J)<\infty$. Then

$$
\nu_{-}(J-T J T)+\nu_{-}(J)=\nu_{-}(J-T)+\nu_{-}(J+T) .
$$

In particular, $\nu_{-}(J-T J T)<\infty$ if and only if $\nu_{-}(J \pm T)<\infty$.

Proof. Consider block operators $\left(\begin{array}{cc}J & T \\ T & J\end{array}\right)$ and $\left(\begin{array}{cc}J+T & 0 \\ 0 & J-T\end{array}\right)$. Equality (5.6) yields $\nu_{-}\left(\begin{array}{cc}J & T \\ T & J\end{array}\right)=\nu_{-}\left(\begin{array}{cc}J+T & 0 \\ 0 & J-T\end{array}\right)$. The negative index of $\left(\begin{array}{cc}J+T & 0 \\ 0 & J-T\end{array}\right)$ equals $\nu_{-}(J-T)+\nu_{-}(J+T)$ and the negative index of $\left(\begin{array}{cc}J & T \\ T & J\end{array}\right)$ is easy to find by using the equality

$$
\left(\begin{array}{cc}
J & T \\
T & J
\end{array}\right)=\left(\begin{array}{cc}
I & 0 \\
T J & I
\end{array}\right)\left(\begin{array}{cc}
J & 0 \\
0 & J-T J T
\end{array}\right)\left(\begin{array}{cc}
I & J T \\
0 & I
\end{array}\right) .
$$

Then one gets (5.7).

Let $\left(\mathfrak{H}_{i},\left(J_{i} \cdot, \cdot\right)\right)(i=1,2)$ and $(\mathfrak{H},(J \cdot, \cdot))$ be Pontryagin spaces, where $\mathfrak{H}=\mathfrak{H}_{1} \oplus \mathfrak{H}_{2}$ and $J=\left(\begin{array}{cc}J_{1} & 0 \\ 0 & J_{2}\end{array}\right)$. Consider an operator $T_{11}=T_{11}^{* *} \in$ 
$\left[\left(\mathfrak{H}_{1}, J_{1}\right)\right]$ such that $\nu_{-}\left[I-T_{11}^{2}\right]=\kappa<\infty$; see $(1.2)$. Denote $\widetilde{T}_{11}=J_{1} T_{11}$, then $\widetilde{T}_{11}=\widetilde{T}_{11}^{*}$ in the Hilbert space $\mathfrak{H}_{1}$. Rewrite

$$
\begin{aligned}
\nu_{-}\left[I-T_{11}^{2}\right] & =\nu_{-}\left(J_{1}\left(I-T_{11}^{2}\right)\right)=\nu_{-}\left(J_{1}-\widetilde{T}_{11} J_{1} \widetilde{T}_{11}\right) \\
& =\nu_{-}\left(\left(J_{1}-\widetilde{T}_{11}\right) J_{1}\left(J_{1}+\widetilde{T}_{11}\right)\right) .
\end{aligned}
$$

Furthermore, denote

$$
\begin{aligned}
& J_{+}=\operatorname{sign}\left(J_{1}\left(I-T_{11}\right)\right)=\operatorname{sign}\left(J_{1}-\widetilde{T}_{11}\right), \\
& J_{-}=\operatorname{sign}\left(J_{1}\left(I+T_{11}\right)\right)=\operatorname{sign}\left(J_{1}+\widetilde{T}_{11}\right), \\
& J_{11}=\operatorname{sign}\left(J_{1}\left(I-T_{11}^{2}\right)\right)
\end{aligned}
$$

and let $\kappa_{+}=\nu_{-}\left[I-T_{11}\right]$ and $\kappa_{-}=\nu_{-}\left[I+T_{11}\right]$. Notice that $\left|I \mp T_{11}\right|=$ $\left|J_{1} \mp \widetilde{T}_{11}\right|$ and one has polar decompositions

$$
I \mp T_{11}=J_{1} J_{ \pm}\left|I \mp T_{11}\right| .
$$

Lemma 5.4. Let $T_{11}=T_{11}^{[*]} \in\left[\left(\mathfrak{H}_{1}, J_{1}\right)\right]$ and $T=\left(\begin{array}{ll}T_{11} & T_{12} \\ T_{21} & T_{22}\end{array}\right) \in[(\mathfrak{H}, J)]$ be a selfadjoint extension of the operator $T_{11}$ with $\nu_{-}\left[I \pm T_{11}\right]<\infty$ and $\nu_{-}(J)<\infty$. Then the following statements

(i) $\nu_{-}\left[I \pm T_{11}\right]=\nu_{-}[I \pm T]$;

(ii) $\nu_{-}\left[I-T^{2}\right]=\nu_{-}\left[I-T_{11}^{2}\right]-\nu_{-}\left(J_{2}\right)$;

(iii) $\operatorname{ran} J_{1} T_{21}^{[*]} \subset \operatorname{ran}\left|I \pm T_{11}\right|^{1 / 2}$

are connected by the implications $(i) \Leftrightarrow(i i) \Rightarrow($ iii $)$.

Proof. The Lemma can be formulated in an equivalent way for the Hilbert space operators: the block operator $\widetilde{T}=J T=\left(\begin{array}{ll}\widetilde{T}_{11} & \widetilde{T}_{12} \\ \widetilde{T}_{21} & \widetilde{T}_{22}\end{array}\right)$ is a selfadjoint extension of $\widetilde{T}_{11}=\widetilde{T}_{11}^{*} \in\left[\mathfrak{H}_{1}\right]$. Then the following statements

(i') $\nu_{-}\left(J_{1} \pm \widetilde{T}_{11}\right)=\nu_{-}(J \pm \widetilde{T})$

(ii') $\nu_{-}(J-\widetilde{T} J \widetilde{T})=\nu_{-}\left(J_{1}-\widetilde{T}_{11} J_{1} \widetilde{T}_{11}\right)-\nu_{-}\left(J_{2}\right)$;

(iii') $\operatorname{ran} \widetilde{T}_{12} \subset \operatorname{ran}\left|J_{1} \pm \widetilde{T}_{11}\right|^{1 / 2}$

are connected by the implications $\left(i^{\prime}\right) \Leftrightarrow\left(i i^{\prime}\right) \Rightarrow\left(i i i^{\prime}\right)$.

Hence it's sufficient to prove this form of the Lemma. 
Let us prove the equivalence $\left(i^{\prime}\right) \Leftrightarrow\left(i i^{\prime}\right)$. Condition (ii') is equivalent to

$$
\nu_{-}\left(\begin{array}{cc}
J_{1} & \widetilde{T}_{11} \\
\widetilde{T}_{11} & J_{1}
\end{array}\right)=\nu_{-}\left(\begin{array}{cc}
J & \widetilde{T} \\
\widetilde{T} & J
\end{array}\right) .
$$

Indeed, in view of (5.8)

$$
\nu_{-}\left(\begin{array}{cc}
J_{1} & \widetilde{T}_{11} \\
\widetilde{T}_{11} & J_{1}
\end{array}\right)=\nu_{-}\left(J_{1}\right)+\nu_{-}\left(J_{1}-\widetilde{T}_{11} J_{1} \widetilde{T}_{11}\right)
$$

and

$$
\begin{aligned}
\nu_{-}\left(\begin{array}{cc}
J & \widetilde{T} \\
\widetilde{T} & J
\end{array}\right) & =\nu_{-}(J)+\nu_{-}(J-\widetilde{T} J \widetilde{T}) \\
& =\nu_{-}\left(J_{1}\right)+\nu_{-}\left(J_{2}\right)+\nu_{-}(J-\widetilde{T} J \widetilde{T}) .
\end{aligned}
$$

By using Lemma 5.3, equality (5.11) is equivalent to

$$
\nu_{-}\left(J_{1}-\widetilde{T}_{11}\right)+\nu_{-}\left(J_{1}+\widetilde{T}_{11}\right)=\nu_{-}(J-\widetilde{T})+\nu_{-}(J+\widetilde{T}) .
$$

Hence, $\left(i^{\prime}\right) \Rightarrow\left(i i^{\prime}\right)$.

Because $\nu_{-}\left(J_{1} \pm \widetilde{T}_{11}\right) \leq \nu_{-}(J \pm \widetilde{T})$, then (5.12) shows that $\left(i i^{\prime}\right) \Rightarrow\left(i^{\prime}\right)$.

Now we prove implication $\left(i i^{\prime}\right) \Rightarrow\left(i i i^{\prime}\right)$; the arguments here will be useful also for the proof of Lemma 5.5 below. Use a permutation to transform the matrix in the right hand side of (5.11):

$$
\nu_{-}\left(\begin{array}{cc}
J & \widetilde{T} \\
\widetilde{T} & J
\end{array}\right)=\nu_{-}\left(\begin{array}{cccc}
J_{1} & 0 & \widetilde{T}_{11} & \widetilde{T}_{12} \\
0 & J_{2} & \widetilde{T}_{21} & \widetilde{T}_{22} \\
\widetilde{T}_{11} & \widetilde{T}_{12} & J_{1} & 0 \\
\widetilde{T}_{21} & \widetilde{T}_{22} & 0 & J_{2}
\end{array}\right)=\nu_{-}\left(\begin{array}{cccc}
J_{1} & \widetilde{T}_{11} & 0 & \widetilde{T}_{12} \\
\widetilde{T}_{11} & J_{1} & \widetilde{T}_{12} & 0 \\
0 & \widetilde{T}_{21} & J_{2} & \widetilde{T}_{22} \\
\widetilde{T}_{21} & 0 & \widetilde{T}_{22} & J_{2}
\end{array}\right) .
$$

Then condition (5.11) implies to the condition

$$
\operatorname{ran}\left(\begin{array}{cc}
0 & \widetilde{T}_{12} \\
\widetilde{T}_{12} & 0
\end{array}\right) \subset \operatorname{ran}\left|\left(\begin{array}{cc}
J_{1} & \widetilde{T}_{11} \\
\widetilde{T}_{11} & J_{1}
\end{array}\right)\right|^{1 / 2} ;
$$

(see Theorem 2.1). By Lemma 5.2 the last inclusion can be rewritten as

$$
\operatorname{ran}\left(\begin{array}{cc}
0 & \widetilde{T}_{12} \\
\widetilde{T}_{12} & 0
\end{array}\right) \subset \operatorname{ran} U\left(\begin{array}{cc}
\left|J_{1}+\widetilde{T}_{11}\right|^{1 / 2} & 0 \\
0 & \left|J_{1}-\widetilde{T}_{11}\right|^{1 / 2}
\end{array}\right) U^{*}
$$


where $U=\frac{1}{\sqrt{2}}\left(\begin{array}{cc}I & I \\ I & -I\end{array}\right)$ is a unitary operator. This inclusion is equivalent to

$$
\begin{aligned}
& \operatorname{ran} U^{*}\left(\begin{array}{cc}
0 & \widetilde{T}_{12} \\
\widetilde{T}_{12} & 0
\end{array}\right) U=\operatorname{ran}\left(\begin{array}{cc}
\widetilde{T}_{12} & 0 \\
0 & -\widetilde{T}_{12}
\end{array}\right) \\
& \subset \operatorname{ran}\left(\begin{array}{cc}
\left|J_{1}+\widetilde{T}_{11}\right|^{1 / 2} & 0 \\
0 & \left|J_{1}-\widetilde{T}_{11}\right|^{1 / 2}
\end{array}\right)
\end{aligned}
$$

and clearly this is equivalent to condition (iii').

Note that if $\widetilde{T}_{11}$ has a selfadjoint extension $\widetilde{T}$ satisfying (i'). Then by applying Theorem 2.1 (or [7, Theorem 1]) it yields (iii').

Lemma 5.5. Let $T_{11}=T_{11}^{[*]} \in\left[\left(\mathfrak{H}_{1}, J_{1}\right)\right]$ be an operator and let

$$
T_{1}=\left(\begin{array}{l}
T_{11} \\
T_{21}
\end{array}\right):\left(\mathfrak{H}_{1}, J_{1}\right) \rightarrow\left(\begin{array}{l}
\left(\mathfrak{H}_{1}, J_{1}\right) \\
\left(\mathfrak{H}_{2}, J_{2}\right)
\end{array}\right)
$$

be an extension of $T_{11}$ with $\nu_{-}\left[I-T_{11}^{2}\right]<\infty, \nu_{-}\left(J_{1}\right)<\infty$, and $\nu_{-}\left(J_{2}\right)<$ $\infty$. Then for the conditions

(i) $\nu_{-}\left[I_{1}-T_{11}^{2}\right]=\nu_{-}\left[I_{1}-T_{1}^{[*]} T_{1}\right]+\nu_{-}\left(J_{2}\right)$;

(ii) $\operatorname{ran} J_{1} T_{21}^{[*]} \subset \operatorname{ran}\left|I-T_{11}^{2}\right|^{1 / 2}$;

(iii) $\operatorname{ran} J_{1} T_{21}^{[*]} \subset \operatorname{ran}\left|I \pm T_{11}\right|^{1 / 2}$

the implications $(i) \Rightarrow($ ii $)$ and $(i) \Rightarrow$ (iii) hold.

Proof. First we prove that (i) $\Rightarrow$ (ii). In fact, this follows from Theorem 3.1 by taking $A=I-T_{11}^{2}$ and $B=T_{21}$.

A proof of (i) $\Rightarrow$ (iii) is quite similar to the proof used in Lemma 5.4. Statement (i) is equivalent the following equation:

$$
\nu_{-}\left(\begin{array}{cc}
J_{1} & \widetilde{T}_{11} \\
\widetilde{T}_{11} & J_{1}
\end{array}\right)=\nu_{-}\left(\begin{array}{cc}
J & \widetilde{T}_{1} \\
\widetilde{T}_{1}^{*} & J_{1}
\end{array}\right) .
$$

Indeed,

$$
\begin{aligned}
& \nu_{-}\left(\begin{array}{cc}
J_{1} & \widetilde{T}_{11} \\
\widetilde{T}_{11} & J_{1}
\end{array}\right)=\nu_{-}\left(\begin{array}{cc}
J_{1} & 0 \\
0 & J_{1}-\widetilde{T}_{11} J_{1} \widetilde{T}_{11}
\end{array}\right) \\
& =\nu_{-}\left(J_{1}-\widetilde{T}_{11} J_{1} \widetilde{T}_{11}\right)+\nu_{-}\left(J_{1}\right)<\infty
\end{aligned}
$$


and

$$
\begin{aligned}
& \nu_{-}\left(\begin{array}{cc}
J & \widetilde{T}_{1} \\
\widetilde{T}_{1}^{*} & J_{1}
\end{array}\right)=\nu_{-}\left(\begin{array}{cc}
J & 0 \\
0 & J_{1}-\widetilde{T}_{1}^{*} J \widetilde{T}_{1}
\end{array}\right) \\
& =\nu_{-}\left(J_{1}-\widetilde{T}_{11} J_{1} \widetilde{T}_{11}-\widetilde{T}_{21}^{*} J_{2} \widetilde{T}_{21}\right)+\nu_{-}\left(J_{1}\right)+\nu_{-}\left(J_{2}\right) .
\end{aligned}
$$

Due to (i) the right hand sides coincide and then the left hand sides coincide as well.

Now let us permutate the matrix in the latter equation.

$$
\nu_{-}\left(\begin{array}{cc}
J & \widetilde{T}_{1} \\
\widetilde{T}_{1}^{*} & J_{1}
\end{array}\right)=\nu_{-}\left(\begin{array}{ccc}
J_{1} & 0 & \widetilde{T}_{11} \\
0 & J_{2} & \widetilde{T}_{21} \\
\widetilde{T}_{11} & \widetilde{T}_{21}^{*} & J_{1}
\end{array}\right)=\nu_{-}\left(\begin{array}{ccc}
J_{1} & \widetilde{T}_{11} & 0 \\
\widetilde{T}_{11} & J_{1} & \widetilde{T}_{21}^{*} \\
0 & \widetilde{T}_{21} & J_{2}
\end{array}\right) .
$$

It follows from [7, Theorem 1] that the condition (i) implies the condition

$$
\begin{aligned}
& \operatorname{ran}\left(\begin{array}{c}
0 \\
\widetilde{T}_{21}^{*}
\end{array}\right) \subset \operatorname{ran}\left|\left(\begin{array}{cc}
J_{1} & \widetilde{T}_{11} \\
\widetilde{T}_{11} & J_{1}
\end{array}\right)\right|^{1 / 2} \\
& =\operatorname{ran} U\left(\begin{array}{cc}
\left|J_{1}+\widetilde{T}_{11}\right|^{1 / 2} & 0 \\
0 & \left|J_{1}-\widetilde{T}_{11}\right|^{1 / 2}
\end{array}\right) U^{*}
\end{aligned}
$$

where $U=\frac{1}{\sqrt{2}}\left(\begin{array}{cc}I & I \\ I & -I\end{array}\right)$ is a unitary operator (see Lemma 5.2). Then, equivalently,

$$
\operatorname{ran} \widetilde{T}_{21}^{*} \subset \operatorname{ran}\left|J_{1} \pm \widetilde{T}_{11}\right|^{1 / 2} .
$$

\subsection{Contractive extensions of contractions with minimal neg- ative indices}

Following to $[7,16,18]$ we consider the problem of existence and a description of selfadjoint operators $T$ in the Pontryagin space $\left(\begin{array}{l}\left(\mathfrak{H}_{1}, J_{1}\right) \\ \left(\mathfrak{H}_{2}, J_{2}\right)\end{array}\right)$ such that $A_{+}=I+T$ and $A_{-}=I-T$ solve the corresponding completion problems

$$
A_{ \pm}^{0}=\left(\begin{array}{cc}
I \pm T_{11} & \pm T_{21}^{[*]} \\
\pm T_{21} & *
\end{array}\right)
$$

under minimal index conditions $\nu_{-}[I+T]=\nu_{-}\left[I+T_{11}\right], \nu_{-}[I-T]=$ $\nu_{-}\left[I-T_{11}\right]$, respectively. Observe, that by Lemma 5.4 the two minimal index conditions above are equivalent to single condition $\nu_{-}\left[I-T^{2}\right]=$ $\nu_{-}\left[I-T_{11}^{2}\right]-\nu_{-}\left(J_{2}\right)$. 
It is clear from Theorem 2.1 that the conditions $\operatorname{ran} J_{1} T_{21}^{[*]} \subset \operatorname{ran} \mid I-$ $\left.T_{11}\right|^{1 / 2}$ and $\operatorname{ran} J_{1} T_{21}^{[*]} \subset \operatorname{ran}\left|I+T_{11}\right|^{1 / 2}$ are necessary for the existence of solutions; however as noted already in [7] they are not sufficient even in the Hilbert space setting.

The next theorem gives a general solvability criterion for the completion problem (5.13) and describes all solutions to this problem. As in the definite case, there are minimal solutions $A_{+}$and $A_{-}$which are connected to two extreme selfadjoint extensions $T$ of

$$
T_{1}=\left(\begin{array}{l}
T_{11} \\
T_{21}
\end{array}\right):\left(\mathfrak{H}_{1}, J_{1}\right) \rightarrow\left(\begin{array}{l}
\left(\mathfrak{H}_{1}, J_{1}\right) \\
\left(\mathfrak{H}_{2}, J_{2}\right)
\end{array}\right)
$$

now with finite negative index $\nu_{-}\left[I-T^{2}\right]=\nu_{-}\left[I-T_{11}^{2}\right]-\nu_{-}\left(J_{2}\right)>$ 0 . The set of solutions $T$ to the problem (5.13) will be denoted by $\operatorname{Ext}_{T_{1}, \kappa}(-1,1)_{J_{2}}$.

Theorem 5.1. Let $T_{1}$ be a symmetric operator as in (5.14) with $T_{11}=$ $T_{11}^{[*]} \in\left[\left(\mathfrak{H}_{1}, J_{1}\right)\right]$ and $\nu_{-}\left[I-T_{11}^{2}\right]=\kappa<\infty$, and let $J_{T_{11}}=\operatorname{sign}\left(J_{1}(I-\right.$ $\left.\left.T_{11}^{2}\right)\right)$. Then the completion problem for $A_{ \pm}^{0}$ in (5.13) has a solution $I \pm T$ for some $T=T^{[*]}$ with $\nu_{-}\left[I-T^{2}\right]=\kappa-\nu_{-}\left(J_{2}\right)$ if and only if the following condition is satisfied:

$$
\nu_{-}\left[I-T_{11}^{2}\right]=\nu_{-}\left[I-T_{1}^{[*]} T_{1}\right]+\nu_{-}\left(J_{2}\right) .
$$

If this condition is satisfied then the following facts hold:

(i) The completion problems for $A_{ \pm}^{0}$ in (5.13) have "minimal" solutions $A_{ \pm}$(for the partial ordering introduced in the first section).

(ii) The operators $T_{m}:=A_{+}-I$ and $T_{M}:=I-A_{-} \in \operatorname{Ext}_{T_{1}, \kappa}(-1,1)_{J_{2}}$.

(iii) The operators $T_{m}$ and $T_{M}$ have the block form

$$
\begin{aligned}
T_{m} & =\left(\begin{array}{cc}
T_{11} & J_{1} D_{T_{11}} V^{*} \\
J_{2} V D_{T_{11}} & -I+J_{2} V\left(I-L_{T}^{*} J_{1}\right) J_{11} V^{*}
\end{array}\right), \\
T_{M} & =\left(\begin{array}{cc}
T_{11} & J_{1} D_{T_{11}} V^{*} \\
J_{2} V D_{T_{11}} & I-J_{2} V\left(I+L_{T}^{*} J_{1}\right) J_{11} V^{*}
\end{array}\right),
\end{aligned}
$$

where $D_{T_{11}}:=\left|I-T_{11}^{2}\right|^{1 / 2}$ and $V$ is given by $V:=\operatorname{clos}\left(J_{2} T_{21} D_{T_{11}}^{[-1]}\right)$.

(iv) The operators $T_{m}$ and $T_{M}$ are "extremal" extensions of $T_{1}$ :

$T \in \operatorname{Ext}_{T_{1}, \kappa}(-1,1)_{J_{2}}$ iff $T=T^{[*]} \in[(\mathfrak{H}, J)], \quad T_{m} \leq_{J_{2}} T \leq_{J_{2}} T_{M}$. 
(v) The operators $T_{m}$ and $T_{M}$ are connected via

$$
(-T)_{m}=-T_{M}, \quad(-T)_{M}=-T_{m}
$$

Proof. It is easy to see by (3.1) that $\kappa=\nu_{-}\left[I-T_{11}^{2}\right] \leq \nu_{-}\left[I-T_{1}^{[*]} T_{1}\right]+$ $\nu_{-}\left(J_{2}\right) \leq \nu_{-}\left[I-T^{2}\right]+\nu_{-}\left(J_{2}\right)$. Hence the condition $\nu_{-}\left[I-T^{2}\right]=\kappa-\nu_{-}\left(J_{2}\right)$ implies (5.15). The sufficiency of this condition is obtained when proving the assertions (i)-(iii) below.

(i) If the condition (5.15) is satisfied then by using Lemma 5.5 one gets the inclusions $\operatorname{ran} J_{1} T_{21}^{[*]} \subset \operatorname{ran}\left|I \pm T_{11}\right|^{1 / 2}$, which by Theorem 2.1 means that each of the completion problems, $A_{ \pm}^{0}$ in (5.13), is solvable. It follows that the operators

$$
S_{-}=\left|I+T_{11}\right|^{[-1 / 2]} J_{1} T_{21}^{[*]}, \quad S_{+}=\left|I-T_{11}\right|^{[-1 / 2]} J_{1} T_{21}^{[*]}
$$

are well defined and they provide the minimal solutions $A_{ \pm}$to the completion problems for $A_{ \pm}^{0}$ in (5.13).

(ii) \& (iii) By Lemma 5.5 the inclusion $\operatorname{ran} J_{1} T_{21}^{[*]} \subset \operatorname{ran}\left|I-T_{11}^{2}\right|^{1 / 2}$ holds. This inclusion is equivalent to the existence of a (unique) bounded operator $V^{*}=D_{T_{11}}^{[-1]} J_{1} T_{21}^{[*]}$ with ker $V \supset \operatorname{ker} D_{T_{11}}$, such that $J_{1} T_{21}^{[*]}=$ $D_{T_{11}} V^{*}$. The operators $T_{m}:=A_{+}-I$ and $T_{M}:=I-A_{-}$(see proof of (i)) by using (5.1), (5.2), and 5.1 can be now rewritten as in (5.16). Indeed, observe that (see Theorem 2.1, (5.9), and (5.10))

$$
\begin{aligned}
J_{2} S_{-}^{*} J_{-} S_{-} & =J_{2} V D_{T_{11}}\left|I+T_{11}\right|^{[-1 / 2]} J_{-}\left|I+T_{11}\right|^{[-1 / 2]} D_{T_{11}} V^{*} \\
& =J_{2} V D_{T_{11}}\left(J_{1}\left(I+T_{11}\right)\right)^{[-1]} D_{T_{11}} V^{*} \\
& =J_{2} V D_{T_{11}} D_{T_{11}}^{[-1]}\left(I+L_{T_{11}}^{*} J_{1}\right)^{[-1]} D_{T_{11}} J_{1} D_{T_{11}} V^{*} \\
& =J_{2} V\left(I+L_{T_{11}}^{*} J_{1}\right)^{[-1]}\left(J_{11}-L_{T_{11}}^{*} J_{T_{11}^{*}} L_{T_{11}}\right) V^{*} \\
& =J_{2} V\left(I+L_{T_{11}}^{*} J_{1}\right)^{[-1]}\left(J_{11}-\left(L_{T_{11}}^{*} J_{1}\right)^{2} J_{11}\right) V^{*} \\
& =J_{2} V\left(I+L_{T_{11}}^{*} J_{1}\right)^{[-1]}\left(I+L_{T_{11}}^{*} J_{1}\right)\left(I-L_{T_{11}}^{*} J_{1}\right) J_{11} V^{*} \\
& =J_{2} V\left(I-L_{T_{11}}^{*} J_{1}\right) J_{11} V^{*},
\end{aligned}
$$

where the third equality follows from (5.1) and the fourth from (5.2). 
And similarly for

$$
\begin{aligned}
J_{2} S_{+}^{*} J_{+} S_{+} & =J_{2} V D_{T_{11}}\left|I-T_{11}\right|^{[-1 / 2]} J_{+}\left|I-T_{11}\right|^{[-1 / 2]} D_{T_{11}} V^{*} \\
& =J_{2} V D_{T_{11}}\left(J_{1}\left(I-T_{11}\right)\right)^{[-1]} D_{T_{11}} V^{*} \\
& =J_{2} V D_{T_{11}} D_{T_{11}}^{[-1]}\left(I-L_{T_{11}}^{*} J_{1}\right)^{[-1]} D_{T_{11}} J_{1} D_{T_{11}} V^{*} \\
& =J_{2} V\left(I-L_{T_{11}}^{*} J_{1}\right)^{[-1]}\left(J_{11}-L_{T_{11}}^{*} J_{T_{11}^{*}} L_{T_{11}}\right) V^{*} \\
& =J_{2} V\left(I-L_{T_{11}}^{*} J_{1}\right)^{[-1]}\left(J_{11}-\left(L_{T_{11}}^{*} J_{1}\right)^{2} J_{11}\right) V^{*} \\
& =J_{2} V\left(I-L_{T_{11}}^{*} J_{1}\right)^{[-1]}\left(I-L_{T_{11}}^{*} J_{1}\right)\left(I+L_{T_{11}}^{*} J_{1}\right) J_{11} V^{*} \\
& =J_{2} V\left(I+L_{T_{11}}^{*} J_{1}\right) J_{11} V^{*},
\end{aligned}
$$

which implies the representations for $T_{m}$ and $T_{M}$ in (5.16). Clearly, $T_{m}$ and $T_{M}$ are selfadjoint extensions of $T_{1}$, which satisfy the equalities

$$
\nu_{-}\left[I+T_{m}\right]=\kappa_{-}, \quad \nu_{-}\left[I-T_{M}\right]=\kappa_{+} .
$$

Moreover, it follows from (5.16) that

$$
T_{M}-T_{m}=\left(\begin{array}{cc}
0 & 0 \\
0 & 2\left(I-J_{2} V J_{11} V^{*}\right)
\end{array}\right) .
$$

Now the assumption (5.15) will be used again. Since $\nu_{-}\left[I-T_{1}^{[*]} T_{1}\right]=$ $\nu_{-}\left[I-T_{11}^{2}\right]-\nu_{-}\left(J_{2}\right)$ and $T_{21}=J_{2} V D_{T_{11}}$ it follows from Theorem 3.1 that $V^{*} \in\left[\mathfrak{H}_{2}, \mathfrak{D}_{T_{11}}\right]$ is $J$-contractive: $J_{2}-V J_{11} V^{*} \geq 0$. Therefore, (5.20) shows that $T_{M} \geq_{J_{2}} T_{m}$ and $I+T_{M} \geq_{J_{2}} I+T_{m}$ and hence, in addition to $I+T_{m}$, also $I+T_{M}$ is a solution to the problem $A_{+}^{0}$ and, in particular, $\nu_{-}\left[I+T_{M}\right]=\kappa_{-}=\nu_{-}\left[I+T_{m}\right]$. Similarly, $I-T_{M} \leq_{J_{2}} I-T_{m}$ which implies that $I-T_{m}$ is also a solution to the problem $A_{-}^{0}$, in particular, $\nu_{-}\left[I-T_{m}\right]=\kappa_{+}=\nu_{-}\left[I-T_{M}\right]$. Now by applying Lemma 5.4 we get

$$
\begin{aligned}
& \nu_{-}\left[I-T_{m}^{2}\right]=\kappa-\nu_{-}\left(J_{2}\right), \\
& \nu_{-}\left[I-T_{M}^{2}\right]=\kappa-\nu_{-}\left(J_{2}\right) .
\end{aligned}
$$

Therefore, $T_{m}, T_{M} \in \operatorname{Ext}_{T_{1}, \kappa}(-1,1)_{J_{2}}$ which in particular proves that the condition (5.15) is sufficient for solvability of the completion problem (5.13).

(iv) Observe, that $T \in \operatorname{Ext}_{T_{1}, \kappa}(-1,1)_{J_{2}}$ if and only if $T=T^{[*]} \supset T_{1}$ and $\nu_{-}[I \pm T]=\kappa_{\mp}$. By Theorem 2.1 this is equivalent to

$$
J_{2} S_{-}^{*} J_{-} S_{-}-I \leq_{J_{2}} T_{22} \leq_{J_{2}} I-J_{2} S_{+}^{*} J_{+} S_{+} .
$$

The inequalities (5.21) are equivalent to (5.17).

(v) The relations (5.18) follow from (5.19) and (5.16). 
Remark 5.1. In case of a contraction operator $T_{1}$ this result coincides with the main result of [16] and in case of a "quasi-contraction" operator $T_{1}$ with finite negative index it coincides with the result of [7, Theorem 5].

\section{References}

[1] N. I. Akhiezer, I. M. Glazman, Theory of Linear Operators in Hilbert Space, Nauka, Moscow, 1966.

[2] T. Ando, K. Nishio, Positive self-adjoint Extensions of Positive Symmetric Operators // Tohóku Math. J., 22 (1970), 65-75.

[3] J. Antezana, G. Corach, and D. Stojanoff, Bilateral shorted operators and parallel sums // Linear Algebra Appl. 414 (2006), 570-588.

[4] Gr. Arsene, A. Gheondea, Completing Matrix Contractions // J. Operator Theory, 7 (1982), 179-189.

[5] Gr. Arsene, T. Constantinescu, A. Gheondea, Lifting of Operators and Prescribed Numbers of Negative Squares // Michigan Math. J., 34 (1987), 201-216.

[6] T. Ya. Azizov, I.S. Iokhvidov, Linear operators in spaces with indefinite metric, John Wiley and Sons, New York, 1989.

[7] D. Baidiuk, S. Hassi, Completion, extension, factorization, and lifting of operators // Math. Ann., 364 (2016), No. 3-4, 1415-1450.

[8] J. Bognár, Indefinite Inner Product Space, Springer-Verlag, Berlin, 1974.

[9] E. A. Coddington, H.S.V. de Snoo, Positive Selfadjoint Extensions of Positive Symmetric Subspaces // Math. Z., 159 (1978), 203-214.

[10] T. Constantinescu, A. Gheondea, Minimal Signature of Lifting operators I // J. Operator Theory, 22 (1989), 345-367.

[11] T. Constantinescu, A. Gheondea Minimal Signature of Lifting operators II // J. Funct. Anal., 103 (1992), 317-352.

[12] Ch. Davis, Some dilation representation theorems // Proceedings of the Second International Symposium in West Africa on Functional Analisys and its Applications, (1979), 159-182.

[13] Ch. Davis, W.M. Kahan, and H.F. Weinberger, Norm preserving dilations and their applications to optimal error bounds // SIAM J. Numer. Anal., 19 (1982), No. 3, 445-469.

[14] M. A. Dritschel, A lifting theorem for bicontractions on Krĕn spaces // J. Funct. Anal., 89 (1990), 61-89.

[15] M. A. Dritschel, J. Rovnyak, Extension theorems for contraction operators on Krein spaces. Extension and interpolation of linear operators and matrix functions, 221-305, Oper. Theory Adv. Appl., 47, Birkhäuser, Basel, 1990.

[16] S. Hassi, M. M. Malamud, and H.S.V. de Snoo, On Krein's Extension Theory of Nonnegative Operators // Math. Nachr., 274/275 (2004), 40-73.

[17] T. Kato, Perturbation Theory for Linear Operators, Springer-Verlag, Berlin, Heidelberg, 1995.

[18] V. U. Kolmanovich, M. M. Malamud, Extensions of Sectorial operators and dual pair of contractions, (Russian) Manuscript No 4428-85. Deposited at Vses. Nauchn-Issled, Inst. Nauchno-Techn. Informatsii, VINITI 1904 85, Moscow, R ZH Mat 10B1144, (1985), 1-57. 
[19] M. G. Kreŭn, Theory of Selfadjoint Extensions of Semibounded Operators and Its Applications, I // Mat. Sb., 20 (1947), No. 3, 431-498.

[20] M. G. Kreĭn, I. E. Ovcharenko, On the Q-functions and sc-resolvents of a nondensely defined Hermitian contraction // Siberian Math. J., 18 (1977), No. 5, 1032-1056.

[21] H. Langer, B. Textorius, Extensions of a bounded Hermitian operator T preserving the numbers of negative eigenvalues of $I-T^{*} T / /$ Research Report LiTH-MATR-87-17, Department of Mathematics, Linköping University, (1977), 15 pp.

[22] M. M. Malamud, On some classes of extensions of a sectorial operators and dual pairs of contractions // Oper. Theory: Adv. Appl., 124 (2001), 401-449.

[23] M. M. Malamud, Operator holes and extensions of sectorial operators and dual pairs of contractions // Math. Nachr., 279 (2006), No. 5-6, 625-655.

[24] B. Sz.-Nagy and C. Foias, Harmonic Analysis of Operators on Hilbert Space, North-Holland Publishing Co., Amsterdam-Budapest, 1970.

[25] S. Parrot, On a quotient norm and the Sz.-Nagy-Foias Lifting Theorem // J. Funct. Anal., 30 (1978), 311-328.

[26] Yu. L. Shmul'yan, A Hellinger operator integral // Mat. Sb. (N.S.), 49 (1959), No. 91, 381-430.

[27] Yu. L. Shmul'yan, R. N. Yanovskaya, Blocks of a contractive operator matrix // Izv. Vyssh. Uchebn. Zaved. Mat., No. 7, (1981), 72-75.

\section{CONTACT INFORMATION}

Dmytro Baidiuk Department of Mathematics and Statistics

University of Vaasa

Vaasa, Finland

E-Mail: dbaidiuk@uwasa.fi 